\title{
Interim recommendations for the reporting of extensively drug resistant and pan-drug resistant isolates of Enterobacteriaceae, Pseudomonas aeruginosa, Acinetobacter spp. and Stenotrophomonas maltophilia
}

German $\mathrm{GJ}^{1}$, Jamieson $\mathrm{FB}^{2}$, Gilmour $\mathrm{M}^{3}$, Almohri $\mathrm{H}^{4}$, Bullard $\mathrm{J}^{5}$, Domingo $\mathrm{MC}^{6}$, Fuller $\mathrm{J}^{7}$, Girouard $\mathrm{G}^{8}$, Haldane $\mathrm{D}^{9}$, Hoang $\mathrm{L}^{10}$, Levett PN ${ }^{11}$, Longtin $\mathrm{J}^{6}$, Melano $\mathrm{R}^{2}$, Needle R ${ }^{12}$, Patel SN ${ }^{2}$, Rebbapragada $A^{13}$, Reyes $\mathrm{RC}^{14}$, and Mulvey $\mathrm{MR}^{3^{*}}$

\section{Note}

The recommendations in this publication should be considered preliminary for one year from the publication date. Comments regarding the document should be sent to Dr. Michael Mulvey. All comments received will be reviewed by the Canadian Public Health Laboratory Network Antimicrobial Resistance Subcommittee before the final recommendations are drafted and released.
Suggested citation: German GJ, Jamieson FB, Gilmour M, Almohri H, Bullard J, Domingo MC, Fuller J, Girouard G, Haldane D, Hoang L, Levett PN, Longtin J, Melano R, Needle R, Patel SN, Rebbapragada A, Reyes $R C$, and Mulvey MR. Interim Recommendations for the Reporting of Extensively Drug Resistant and Pan Drug Resistant Isolates of Enterobacteriaceae, Pseudomonas aeruginosa, Acinetobacter spp. and Stenotrophomonas maltophilia. Can Comm Dis Rep 2016;42:91-7. https://doi.org/10.14745/ccdr.v42i04a04

\author{
Affiliations \\ ${ }^{1}$ Health PEI, Charlottetown, PEI \\ ${ }^{2}$ Public Health Ontario Laboratories, \\ Toronto, ON \\ ${ }^{3}$ National Microbiology Laboratory, \\ Public Health Agency of Canada, \\ Winnipeg, MB \\ ${ }^{4}$ LifeLabs, Toronto, ON \\ ${ }^{5}$ Cadham Provincial Laboratory, \\ Winnipeg, MB \\ ¿Laboratoire de santé \\ publique du Québec, INSPQ, \\ Ste-Anne-de-Bellevue, QC \\ ${ }^{7}$ Alberta Provincial Laboratory for \\ Public Health, Edmonton, AB \\ ${ }^{8}$ Centre hospitalier universitaire \\ Dr-Georges-L.-Dumont, Moncton, NB \\ ${ }^{9}$ Queen Elizabeth II Health Science \\ Centre, Halifax, NS \\ ${ }^{10} \mathrm{BC}$ Centre for Disease Control Public \\ Health Laboratory, Vancouver, BC \\ ${ }^{11}$ Saskatchewan Disease Control \\ Laboratory, Regina, SK \\ ${ }^{12}$ Newfoundland Public Health \\ Laboratory, St. John's, NL \\ ${ }^{13}$ Dynacare, Brampton, ON \\ ${ }^{14}$ LifeLabs, Burnaby, BC \\ *Correspondence: Michael. \\ Mulvey@phac-aspc.gc.ca
}

\subsection{Introduction}

These recommendations are produced under the auspices and authority of the Canadian Public Health Laboratory Network, Antimicrobial Resistance Working Group. They represent a consensus of peer reviewed information and expert opinion on the most appropriate ways to test for and report a multi-drug resistant phenotype in common Gram-negative pathogens.

These recommendations were developed for use by all Canadian non-veterinary clinical microbiology laboratories to provide standardization for provincial and national surveillance programs.

\subsection{Background}

Antimicrobial resistance is a growing concern for human health as bacterial pathogens continue to accumulate genes and genomic alterations that confer resistance to antimicrobials. Most concerning is the occurrence of multiple resistance traits within individual key pathogens, which greatly limits, if not entirely eliminates the arsenal of effective treatment options for those infections, thereby leading to poor clinical outcomes. In Canada, we have observed these highly resistant strains in Enterobacteriaceae, Acinetobacter spp., Stenotrophomonas 
maltophilia, and Pseudomonas aeruginosa (1-3). There is a need for laboratories to classify organisms that are resistant to multiple antimicrobials in order to consistently and accurately share the information locally, nationally, and internationally with the medical community, public health authorities and policy makers. More specifically, classification as 'multi-drug resistant' is commonly an actionable finding within hospital Infection Prevention \& Control programs. Recently, there has been a proposal to internationally standardize these definitions in selected Gram-positive and Gram-negative organisms (4), yet this proposal for interim definitions has not yet led to a revised definition or national recommendations.

The goal of this document is to provide Canadian laboratories with a framework for consistent reporting and monitoring of multi-drug resistant organisms (MDRO), extensively drug resistant organisms (XDRO), and pan-drug resistant organisms (PDRO). The recommendations were based on an interim international proposal published in 2012 for Gram-negative organisms (4). This document modifies the following for the Canadian setting: 1) Resistance was used instead of non-susceptibility (Intermediate and Resistant) to better match which antimicrobials will be clinically used for treating resistant infections; antimicrobials that are more easily tested in the laboratory; and those that would limit unnecessary reference testing. 2) MDRO rules are separated for commonly used antimicrobials in the community setting for urine infections and non-urine infections. 3) Rather than all classes of antimicrobials being considered in the definitions, only relevant classes that are commonly tested in Canadian clinical laboratories were considered. Also within a class of antimicrobials, resistance to the most commonly used antimicrobial for treating severe infections (i.e. meropenem or imipenem) was considered rather than an inferior drug for infections (i.e. ertapenem for the carbapenems). 4) Since XDRO definitions will fluctuate from country to country based on $2^{\text {nd }}$ and $3^{\text {rd }}$ line available antimicrobials, adjustments were made for antimicrobials available/approved for use in Canada rather than all drug categories listed in the Clinical Laboratory Standards Institute (CLSI) (5). The justification for these modifications can be found in Appendix 1. Over time as new antimicrobials become available, previously available antimicrobials lose effectiveness, or no longer available, the definitions will necessitate periodic review. The recommendations stated herein are considered interim and are open for stakeholder consultation such that future recommendations evolve to accommodate public health, community care, and acute care partners.

\subsection{Recommendations for Antimicrobial Susceptibility Testing}

3.1 A resistant interpretation of an isolate can be determined using disk diffusion, broth microdilution, or agar dilution following CLSI guidelines for the testing of Enterobacteriaceae, Pseudomonas aeruginosa, Acinetobacter spp. and

Stenotrophomonas maltophilia (5). A Health Canada or Federal Drug Administration (FDA) approved automated method or gradient diffusion strips can also be used for the generation of the antimicrobial susceptibility data.

3.2 Current CLSI breakpoints (M100) for resistance should be used when determining the designations of MDRO,
XDRO, and PDRO. It is understood that some laboratories use automated methods with Food and Drug Administration (FDA; www.fda.gov) breakpoints that may differ from the CLSI recommendations. A laboratory using FDA breakpoints should include the breakpoint difference in any report for MDRO, $\mathrm{XDRO}$, and PDRO.

3.3 Certain species of Enterobacteriaceae should not be tested for particular antimicrobial agents because of intrinsic resistance to the agent (Table 1, Exceptions).

\subsection{Definitions of Screening/Testing for MDRO, XDRO and PDRO}

These interim recommendations are to be applied only to clinical/diagnostic specimens. However, acute care and long term care facilities, and by extension health authorities, may choose to still apply the definitions of MDRO/XDRO/PDRO for screening purposes as determined by their own fiscal situation and local health resources. If isolates are part of a specialized surveillance program (e.g. in-patient screening), it should be clearly indicated in the laboratory report that the MDRO/XDRO/ $\mathrm{PDRO}$ is pertinent for colonization or carriage status only.

\subsection{Enterobacteriaceae Multi-Drug Resistance Definition}

It is recognized that laboratories may not test Gram-negative isolates for all classes of antimicrobial agents and therefore would not be able to determine MDRO, XDRO, and PDRO. Therefore, we have included a category of multi-drug resistant organisms (MDRO) that should be considered for screening isolates for XDRO or PDRO.

4.1.1 There are four rules for MDRO status in Enterobacteriaceae which takes into consideration the specific specimen type (Table 1).

\subsection{Acinetobacter spp. or Pseudomonas aeruginosa Multi-drug Resistance Definition}

4.2.1 An isolate should be considered MDRO if resistant to THREE of the FIVE antimicrobial agents listed below (Table 2):

1. Ciprofloxacin

2. Piperacillin-tazobactam OR piperacillin (specifically for $P$. aeruginosa)

3. Ceftazidime OR cefepime

4. Imipenem OR meropenem

5. Tobramycin

\subsection{Stenotrophomonas maltophilia Multi-Drug Resistance Definition}

4.3.1 S. maltophilia is intrinsically resistant to all carbapenems and most cephalosporins. A clinical isolate should be considered an MDRO if it is resistant to trimethoprim-sulfamethoxazole and subsequent susceptibility testing indicates it is also resistant to an oral anti-microbial (minocycline or levofloxacin) [Table 2]. 
Table 1: Rules for the determination of Multi-Drug-, Extensively Drug-, Pan-Drug Resistant Organisms in Enterobacteriaceae from clinical isolates ${ }^{\mathrm{a}}$

\begin{tabular}{|c|c|c|c|}
\hline Rule & Speciman & Antimicrobial Groups & Interpretation \\
\hline \multirow[t]{4}{*}{1} & \multirow[t]{4}{*}{ Urine } & $\begin{array}{l}\text { Cefixime OR } \\
\text { Amoxicillin-clavulanate }\end{array}$ & \multirow{4}{*}{$\begin{array}{l}\text { Resistance to } \\
\text { THREE of the } \\
\text { FOUR groups = } \\
\text { MDRO }\end{array}$} \\
\hline & & Ciprofloxacin & \\
\hline & & Trimethoprim-Sulfamethoxazole & \\
\hline & & Nitrofurantoin & \\
\hline \multirow[t]{3}{*}{2} & \multirow[t]{3}{*}{ Non-Urine } & $\begin{array}{l}\text { (Cefixime OR } \\
\text { Amoxicillin-clavulanate) }\end{array}$ & \multirow{3}{*}{$\begin{array}{l}\text { Resistance to } \\
\text { THREE of the } \\
\text { THREE groups = } \\
\text { MDRO }\end{array}$} \\
\hline & & Ciprofloxacin & \\
\hline & & Trimethoprim-sulfamethoxazole & \\
\hline 3 & All & $\begin{array}{l}\text { Meropenem } \\
\text { AND } \\
\text { (Ciprofloxacin } \\
\text { OR } \\
\text { Trimethoprim-sulfamethoxazole) }\end{array}$ & $\begin{array}{l}\text { Resistance to } \\
\text { a very broad } \\
\text { spectrum } \\
\text { antimicrobial and } \\
\text { resistance to one } \\
\text { of two commonly } \\
\text { used and } \\
\text { unrelated drug } \\
\text { classes = MDRO }\end{array}$ \\
\hline 4 & All & $\begin{array}{l}\text { Tobramycin } \\
\text { AND } \\
\text { Gentamicin } \\
\text { AND } \\
\text { Piperacillin-Tazobactam } \\
\text { AND } \\
\text { (Ciprofloxacin } \\
\text { OR } \\
\text { Trimethoprim-sulfamethoxazole) }\end{array}$ & $\begin{array}{l}\text { Resistance to } \\
\text { two commonly } \\
\text { susceptible } \\
\text { drug classes and } \\
\text { resistance to one } \\
\text { of two commonly } \\
\text { used and } \\
\text { unrelated drug } \\
\text { classes = MDRO }\end{array}$ \\
\hline \multirow[t]{6}{*}{5} & \multirow[t]{6}{*}{ All } & Tobramycin AND Gentamicin & \multirow{6}{*}{$\begin{array}{l}\text { Resistance to } \\
\text { FOUR of } \\
\text { the SIX } \\
\text { antimicrobial } \\
\text { groups = XDRO }\end{array}$} \\
\hline & & Piperacillin-Tazobactam & \\
\hline & & Imipenem OR Meropenem & \\
\hline & & $\begin{array}{l}\text { Cefepime OR (cefotaxime/ } \\
\text { ceftriaxone) AND ceftazidime }\end{array}$ & \\
\hline & & Ciprofloxacin & \\
\hline & & Trimethoprim-Sulfamethoxazole & \\
\hline 6 & All & Same groups listed in rule \#5 & $\begin{array}{l}\text { Resistance } \\
\text { to SIX of SIX } \\
\text { antimicrobial } \\
\text { groups = PDRO }\end{array}$ \\
\hline
\end{tabular}

Abbreviations: MDRO, multi-drug resistant organisms; XDRO, extensively drug resistant organisms; PDRO, pan-drug resistant organisms

a Expert rules modified from Leclercq et al., 2013 (7)

b Imipenem can be substituted for meropenem with the exception of Proteus spp.

\subsection{Confirmation of XDRO}

\subsection{Enterobacteriaceae XDRO Definition}

5.1.1 An isolate that has been determined to be an MDRO should be considered an XDRO by testing/assessing resistance to other antimicrobial agents listed in this section.
5.1.2 Unlike the definition of MDRO for Enterobacteriaceae, the type of specimen does not need to be considered for the definition of XDRO.

5.1.3 An isolate of Enterobacteriaceae should be considered an XDRO when the isolate is resistant to FOUR of the $\underline{\text { SIX }}$ antimicrobial agents listed below (Table 1):

1. Tobramycin AND gentamicin

2. Piperacillin-tazobactam

3. Imipenem OR meropenem

4. Cefepime OR (cefotaxime/ceftriaxone) AND ceftazidime

5. Ciprofloxacin

6. Trimethoprim-sulfamethoxazole

\subsection{Pseudomonas aeruginosa XDRO Definition}

5.2.1 A P. aeruginosa should be considered an XDRO when the isolate is resistant to FOUR of the SIX antimicrobial agents listed below (Table 2):

1. Tobramycin

2. Piperacillin OR piperacillin-tazobactam

3. Imipenem OR meropenem OR doripenem

4. Cefepime OR ceftazidime

5. Ciprofloxacin

6. Colistin

5.2.2 A P. aeruginosa should be considered a PDRO when the isolate is resistant to ALL of the antimicrobial agents listed in 5.2.1.

\subsection{Acinetobacter spp. XDRO Definition}

5.3.1 An Acinetobacter spp. should be considered an XDRO when the isolate is resistant to $\underline{\mathrm{SIX}}$ of the EIGHT antimicrobial agents listed below (Table 2):

1. Gentamicin OR Tobramycin

2. Piperacillin-tazobactam

3. Imipenem OR meropenem OR doripenem

4. Cefepime OR ceftazidime

5. Ciprofloxacin

6. Colistin

7. Doxycycline OR minocycline

8. Trimethoprim-sulfamethoxazole (note: intrinsically resistant to trimethoprim)

\subsection{Stenotrophomonas maltophilia XDRO Definition}

A S. maltophilia should be considered an XDRO if resistant to three oral antimicrobials (trimethoprim-sulfamethoxazole, minocycline, and levofloxacin). The isolate should be referred for complete antimicrobial susceptibility testing to exclude a PDRO (see Table 2). 


\subsection{Confirmation of PDRO}

An Enterobacteriaceae, P. aeruginosa, Acinetobacter spp. should be considered a PDRO when the isolate is resistant to ALL antimicrobial agents listed in Table 1 (rule 6), section 5.2.1, or 5.3.1, respectively. S. maltophilia should be considered a PDRO if it is resistant to all of the following: trimethoprim-sulfamethoxazole, levofloxacin, ceftazidime, and chloramphenicol.

\subsection{Reporting to Reference Laboratories}

7.1 Any laboratory identifying a MDRO that cannot confirm an XDRO or PDRO using additional antimicrobial susceptibility tests should send the isolate to a reference (provincial) laboratory (See Appendix 2).

7.2 The reference (provincial) laboratory should be notified of any XDR or PDR organisms identified and the isolate should be forwarded to the reference laboratory, and should include the following information:

1. Age of patient
2. Gender of patient

3. Type of clinical specimen (blood, respiratory, skin/soft tissue, or urine)

4. Date of collection

5. Antimicrobial susceptibility testing results from submitting laboratory

6. Out of Canada travel history in the last 3 months. Travel history is dated from the time of the first isolation of the organism. This is highly recommended for inpatients and desirable for outpatients. All countries traveled should be listed.

7.3 If multiple clinical isolates of the same species and susceptibility pattern are recovered from the same patient, send the isolate from the most invasive site where possible. Additional isolates of the same species and susceptibility pattern should be reported/sent to a reference laboratory no more frequently than every 7 days after the first isolate. Annotating as an MDRO/ XDRO/PDRO on the clinical report should continue for each isolate regardless number of isolates or time interval between specimens.

Table 2: Definitions for the determination of Multi-Drug-, Extensively Drug-, Pan-Drug Resistant Organisms in select organisms

\begin{tabular}{|c|c|c|c|}
\hline \multicolumn{2}{|r|}{ MDRO } & \multicolumn{2}{|c|}{ XDRO / PDRO } \\
\hline Definition & Antimicrobial Groups & Definitions & Antimicrobial Groups \\
\hline \multicolumn{4}{|c|}{ Organism: Pseudomonas aeruginosa } \\
\hline \multirow{7}{*}{$\begin{array}{l}\text { Resistance to } \\
\text { THREE of the FIVE } \\
\text { antimicrobial groups }\end{array}$} & Ciprofloxacin & \multirow{3}{*}{$\begin{array}{l}\text { Resistance to FOUR of the } \underline{\text { SIX }} \\
\text { antimicrobial groups }=\text { XDRO }\end{array}$} & Tobramycin \\
\hline & Piperacillin-tazobactam OR piperacillin & & Piperacillin-tazobactam OR piperacillin \\
\hline & Ceftazidime OR cefepime & & Imipenem OR meropenem OR \\
\hline & Imipenem OR meropenem & \multirow{4}{*}{$\begin{array}{l}\text { Resistance to } \underline{\mathrm{SIX}} \text { of the } \underline{\mathrm{SIX}} \\
\text { antimicrobial groups = PDRO }\end{array}$} & doripenem \\
\hline & \multirow{3}{*}{ Tobramycin } & & Cefepime OR ceftazidime \\
\hline & & & Ciprofloxacin \\
\hline & & & Colistin \\
\hline \multicolumn{4}{|c|}{ Organism: Acinetobacter spp. } \\
\hline \multirow{9}{*}{$\begin{array}{l}\text { Resistance to } \\
\text { THREE of the FIVE } \\
\text { antimicrobial groups }\end{array}$} & Ciprofloxacin & \multirow{9}{*}{$\begin{array}{l}\text { Resistance to } \underline{\text { SIX of the EIGHT }} \\
\text { antimicrobial groups = XDRO } \\
\text { Resistance to all groups = PDRO }\end{array}$} & Gentamicin OR tobramycin \\
\hline & Piperacillin-tazobactam & & \\
\hline & Ceftazidime OR cefepime & & Piperacillin-tazobactam \\
\hline & Imipenem OR meropenem & & $\begin{array}{l}\text { Imipenem OR meropenem OR } \\
\text { doripenem }\end{array}$ \\
\hline & \multirow[t]{5}{*}{ Tobramycin } & & Cefepime OR ceftazidime \\
\hline & & & Ciprofloxacin \\
\hline & & & Colistin \\
\hline & & & Doxycycline OR minocycline \\
\hline & & & Trimethoprim-sulfamethoxazole \\
\hline \multicolumn{4}{|c|}{ Organism: Stenotrophomonas maltophilia } \\
\hline \multirow{5}{*}{$\begin{array}{l}\text { Resistance to } \underline{\text { BOTH }} \\
\text { antimicrobial groups }\end{array}$} & \multirow{5}{*}{ Trimethoprim-sulfamethoxazole } & \multirow{2}{*}{$\begin{array}{l}\text { Resistance to the FIRST THREE } \\
\text { antimicrobial groups = XDRO }\end{array}$} & Trimethoprim-sulfamethoxazole \\
\hline & & & Minocycline \\
\hline & & & Levofloxacin \\
\hline & & $\begin{array}{l}\text { Resistance to all antımıcrobial } \\
\text { groups = PDRO }\end{array}$ & Ceftazidime \\
\hline & & & Chloramphenicol \\
\hline
\end{tabular}

Abbreviations: MRDO, multi-drug resistant organsims; XDRO, extensively drug resistant organisms; PDRO, pan-drug resistant organisms 
7.4 It is suggested that reports of clinical specimens found to contain XDRO or PDRO isolates incorporate the term Extensively Drug Resistant Organism or Pan-Drug Resistant Organism within the body of the clinical report.

7.5 Any XDRO or PDRO isolate identified should be reported to public health according to local, regional, and provincial regulations with the additional information outlined in 7.2.

7.6 The originating laboratory should retain the XDRO or PDRO isolates for at least six months, or as required by provincial or local regulations.

7.7 The reference (provincial) laboratory should report all of the data to the National Microbiology Laboratory as defined in 7.2.

\section{Acknowledgements}

The subcommittee would like to acknowledge the work of Dr. John Conly (University of Alberta), Dr. Charles Frenette (McGill University), and all the other members of the Canadian Infectious Disease Steering Committee Antimicrobial Resistance Surveillance Task Group. We also appreciate the support of Dr. George Zhanel (University of Manitoba) of the Canadian Antimicrobial Resistance Alliance for feedback on earlier versions of the document. We thank members of the Canadian Public Health Laboratory Network Laboratory Director's Council for review and final approval of the document. We would also like to thank Ms. Sandra Radons-Arneson from our secretariat for her support.

\section{Conflict of interest}

None.

\section{Funding}

This work was supported in kind by all laboratories of the authors and the Canadian Public Health Laboratory Network Antimicrobial Resistance Subcommittee.

\section{References}

1. Laupland KB, Parkins MD, Church DL, Gregson DB, Louie TJ, Conly JM, Elsayed S, Pitout JDD. 2005. Population-based epidemiological study of infections caused by carbapenemresistant Pseudomonas aeruginosa in the Calgary Health Region: importance of metallo-beta-lactamase (MBL)producing strains. J Infect Dis 192:1606-1612.
2. Mataseje LF, Bryce E, Roscoe D, Boyd DA, Embree J, Gravel D, Katz K, Kibsey P, Kuhn M, Mounchili A, Simor A, Taylor G, Thomas E, Turgeon N, Mulvey MR, Canadian Nosocomial Infection Surveillance Program. 2012. Carbapenem-resistant Gram-negative bacilli in Canada 2009-10: results from the Canadian Nosocomial Infection Surveillance Program (CNISP). J Antimicrob Chemother 67:1359-1367.

3. Tien HC, Battad A, Bryce EA, Fuller J, Mulvey M, Bernard K, Brisebois R, Doucet JJ, Rizoli SB, Fowler R, Simor A. 2007. Multi-drug resistant Acinetobacter infections in critically injured Canadian forces soldiers. BMC Infect Dis 7:95-2000.

4. Magiorakos AP, Srinivasan A, Carey RB, Carmeli Y, Falagas ME, Giske CG, Harbarth S, Hindler JF, Kahlmeter G, OlssonLiljequist B, Paterson DL, Rice LB, Stelling J, Struelens MJ, Vatopoulos A, Weber JT, Monnet DL. 2012. Multidrugresistant, extensively drug-resistant and pandrug-resistant bacteria: an international expert proposal for interim standard definitions for acquired resistance. Clin Microbiol Infect 18:268-281.

5. Clinical and Laboratory Standards Institute. Performance Standards for Antimicrobial Susceptibility Testing: TwentyThird Informational Supplement M100-S25. CLSI, Wayne, PA, USA, 2015.

6. Mosby's Medical Dictionary 9th ed 2012 St. Louis, MO: Mosby Elsevier.

7. Leclercq R, Canton R, Brown DFJ, Giske CG, Heisig P, MacGowan AP, Mouton JW, Nordmann P, Rodloff AC, Rossolini GM, Soussy CJ, Steinbakk M, Winstanley TG, Kahlmeter G. 2013. EUCAST expert rules in antimicrobial susceptibility testing. Clin Microbiol Infect 19:141-160. 


\section{Appendix 1}

\section{Methodology for Developing the Recommendations}

The article published by Magiorakos and colleagues (2012) was used as the main reference for the development of these Canadian recommendations. Drs. German and Mulvey developed the initial framework for the document, which was reviewed by the Canadian Public Health Laboratory Network (CPHLN) AMR Working Group members and invited collaborators. Two main considerations were discussed by the working group members: (i) formulation of a recommendation that focused on antimicrobial drugs commonly used in Canada; and (ii) creation of a document that is easy to use by frontline laboratories, which predominantly utilize automated methods for generating antimicrobial susceptibility data.

Three rounds of discussion and document revision took place with the working group. This included discussion and suggestions from the Communicable and Infectious Disease Steering Committee (CIDSC) AMR Task Group from the Pan-Canadian Public Health Network. The final draft recommendations were reviewed by the CPHLN Executive.

Major variation with recommendations in this document as compared to Magiorakos et. al. (2012) was as follows:

1. The working group decided to focus on Gram-negative isolates to keep the recommendations straightforward and achievable. It was decided that recommendations for Gram-positive organisms would be addressed in a future document;

2. Stenotrophomonas maltophilia was added as an additional Gram-negative organism to be considered for the reporting of MDRO, XDRO and PDRO in the Canadian document;

3. Although the definition of MDRO in Gram-negative organisms is an important consideration, given the treatment complications that can be associated with these infections, it was decided at a provincial and national level to voluntarily report only XDRO and PDRO isolates and use the identification of an MDRO as a screening test to direct further testing and reporting of resistant isolates. This was done to ensure frontline laboratories could easily report their findings to reference laboratories, or request additional tests of antimicrobial drugs not covered under the frontline antimicrobial drug panel needed to confirm XDRO/PDRO.
4. A great deal of discussion focused on the value of using the definition of resistance, as defined by CLSI (2015), rather than that of non-susceptibility proposed by Magiorakos et. al. (2012). It was decided to use the CLSI definition of resistance based on the main arguments put forward, which were: (i) front-line laboratories may have difficulty analyzing 'intermediate resistance' data in the context of MDRO/ XDRO/PDRO; (ii) there were concerns about the reporting of these organisms in relation to public health. A stringent definition of resistance was determined to be the most feasible solution.

5. It was noted that laboratories may have to use FDA breakpoints, which may differ from the CLSI definitions. It was requested in the recommendations that these differences be noted in the report to the reference laboratory.

6. The exhaustive antimicrobial agents listed in the Tables of the Magiorakos et. al. (2012) publication was simplified to reflect the antimicrobial agents commonly used and available in Canada.

7. Ertapenem was removed as a marker for carbapenem resistance in Enterobacteriaceae. The specificity of ertapennem is lower than that of meropenem and imipenem and is not commonly used in a clinical laboratory setting.

8. With the exception of Acinetobacter spp. and S. maltophilia, the tetracyclines were removed from the list of antimicrobials to be considered as they are not frequently tested in frontline laboratories nor are they commonly used to treat serious infections.

9. The Canadian recommendations requested additional clinical information that were not included in the Magiorakos et. al. (2012) publication. 


\section{Appendix 2}

\section{Reference Laboratory Contact Information}

Dr. Linda Hoang

BC Public Health Microbiology \& Reference Laboratory, 655 West 12th Avenue,

Vancouver, BC, V5Z 4R4

linda.hoang@bccdc.ca

Dr. Jeff Fuller

Alberta Provincial Laboratory for Public Health, Alberta Health Services,

2B3.13 WMC, 8440-112 Street,

Edmonton, AB, T6G 2J2

jeff.fuller@albertahealthservices.ca

Dr. Paul Levett

Saskatchewan Disease Control Laboratory,

5 Research Drive,

Regina, SK, S4S 0A4

plevett@health.gov.sk.ca

Dr. Jared Bullard

Cadham Provincial Laboratory,

750 William Avenue,

Winnipeg, MB, R3E 3J7

jared.bullard@gov.mb.ca

Dr. Samir Patel

Public Health Ontario Laboratories,

661 University Avenue, Ste. 1701

Toronto, ON, N5G 1M1

samir.patel@oahpp.ca

Dr. Brigitte Lefebvre

Laboratoire de santé publique du Québec,

Institut national de santé publique du Québec, 20045 chemin Sainte-Marie,

Ste-Anne-de-Bellevue (Québec) H9X 3R5

marc-christian.domingo@inspq.qc.ca
Dr. Gabriel Girouard

Centre Hospitalier Universitaire Dr-Georges-L-Dumont, 330 Avenue Universite,

Moncton, NB, E1C $2 Z 3$

gabriel.girouard@vitalitenb.ca

Dr. David Haldane

Queen Elizabeth II Health Science Centre,

5788 University Avenue,

Halifax, NS, B3H 1 V8

david.haldane@cdha.nshealth.ca

Dr. Greg German

Queen Elizabeth Hospital

60 Riverside Drive

Charlottetown, PE C1A 8T5

gjgerman@ihis.org

Robert Needle

Newfoundland Public Health Laboratory,

Dr. L.A. Miller Centre,

100 Forest Road,

St. John's, NL, A1A $3 Z 9$

robert.needle@easternhealth.ca

Dr. Michael Mulvey

National Microbiology Laboratory

1015 Arlington Street

Winnipeg, MB, R3E 3R2

michael.mulvey@phac-aspc.gc.ca 